\title{
A Cyclostationarity Analysis Applied to Image Forensics
}

\author{
Babak Mahdian and Stanislav Saic \\ Institute of Information Theory and Automation of the ASCR \\ Pod Vodárenskou věží 4, 18208 Prague 8, Czech Republic \\ mahdianeutia.cas.cz \\ ssaic@utia.cas.cz
}

\begin{abstract}
The processing history of images plays an important role in many fields of digital image processing and computer vision. In this paper, we focus on geometrical transformations and show that images that have undergone such transformations contain hidden cyclostationary features. This makes possible employing the well-developed theory and efficient methods of cyclostationarity for blind analyzing of history of images in respect to geometrical transformations. To verify this, we also propose a cyclostationarity detection method and show how the traces of geometrical transformations in an image can be detected and the specific parameters of the transformation estimated. The method is based on the fact that a cyclostationary signal has a frequency spectrum correlated with a shifted version of itself.
\end{abstract}

\section{Introduction}

The knowledge of image's geometric history plays an important role in image compression, image registration, medical image analysis, image retrieval, digital publishing, etc. Furthermore, it has a crucial role in image forensics. Here the detection of traces of geometrical transformations signifies photo manipulation (for example, see $[13,9,6]$ ). Moreover, when applying a statistical-based method to an image, without knowing the processing history of this image and how the statistics of the image has been changed, we can expect miscalculations and unexpected results. The core of the paper is based on the cyclostationarity theory, which is an attractive and novel one for the computer vision and pattern recognition community.

The term cyclostationarity refers to a special class of signals which exhibit periodicity in their statistics. In this paper, we focus on geometrical transformations and show that images that have undergone such transformations contain hidden cyclostationary features. This will justify employing the well-developed theory of cyclostationarity and its efficient methods for analyzing images' history in respect to geometrical transformations. We analytically show that the cyclostationarity is brought into the signal by the interpolation process (nearest neighbor, linear, cubic, etc.). The interpolation process is present in almost every image resizing or rotation operation. Interpolation has a long history and probably started being used as early as 2000BC by ancient Babylonian mathematicians ${ }^{1}$. Despite this long history, the massive usage of interpolation and its importance in digital signal processing, to our knowledge, there exist only a few published works concerned with the specific changes brought into the signal by this process $[13,10,7,1]$. The knowledge that interpolated/resampled images are defacto cyclostationary signals makes possible a new point of view to such images and justifies employing existing efficient cyclostationarity detectors to improve the results of mentioned methods.

One of the most important properties of a cyclostationary signal is the existence of specific correlation between its spectral components [4]. Based on this knowledge, we also propose a cyclostationarity detection method capable of determining whether a given digital image is a result of a geometric transformation. If so, the method also can determine the specific parameters of transformation.

Comparing the method described in this paper with [13] shows, that the latter one is based on a complex and timeconsuming expectation-maximization algorithm (EM). Our method uses a simple and fast method for detecting cyclostationary features and achieves very similar results. Furthermore, the output of the method in [13] and the convergency of their EM part directly depend on several initialization parameters. Our method does not need any parameters initialization and work in a complete blind way.

\section{Cyclostationarity}

In the last half a century a lot of work has been done in the field of cylcostationarity [5]. Much of the initial work introducing and examining the use of cyclostationary mod-

\footnotetext{
${ }^{1}$ For instance, it had an important role in astronomy which in those days was all about time-keeping and making predictions concerning astronomical events [11].
} 
els in the signal analysis was carried out by W. A. Gardner et al. [3, 4, 2].

A zero-mean signal $f(x)$ is defined to be second order cyclostationary if its second order statistics is periodic. The autocorrelation function of $f(x)$ can be defined as

$$
R_{f}(x, \delta)=E\left\{f(x) f^{*}(x+\delta)\right\}
$$

because of its periodicity in $x$, we can represent it in the form of a Fourier series expansion:

$$
R_{f}(x, \delta)=\sum_{\alpha} R_{f}^{\alpha}(\delta) e^{j 2 \pi \alpha x}
$$

where $\alpha$ is the cyclic frequency. The parameter $R_{f}^{\alpha}$ is called Cyclic Autocorrelation Function (CAF) and it is a fundamental parameter of cyclostationarity. CAF is defined as:

$$
R_{f}^{\alpha}(\delta)=\lim _{X \rightarrow \infty} \frac{1}{X} \int_{-X / 2}^{X / 2} R_{f}(x, \delta) e^{-j 2 \pi \alpha x} d x
$$

An appropriate way of analyzing cyclostationary properties is by applying the Fourier Transform (FT) to $R_{f}^{\alpha}$. The result is called Spectral Correlation Function (SCF) and is defined as:

$$
S_{f}^{\alpha}(u)=\int_{-\infty}^{\infty} R_{f}^{\alpha}(\delta) e^{-j 2 \pi u \delta} d \delta
$$

As we will deal with discrete signals, the discrete version of CAF should also be defined here:

$$
R_{f}^{\alpha}(l)=\lim _{N \rightarrow \infty} \frac{1}{N} \sum_{m=0}^{N-1} f[m] f^{*}[m+l] e^{-j 2 \pi \alpha m \Delta m}
$$

where $N$ and $\Delta m$ denote the number of samples of the signal and sampling interval, respectively. Equivalently, the discrete SCF can be obtained by:

$$
S_{f}^{\alpha}(u)=\sum_{l=-\infty}^{\infty} R_{f}^{\alpha}(l) e^{-j 2 \pi u l \Delta l}
$$

CAF and SCF are analogous to the autocorrelation function and power spectral density function for stationary signals. When $\alpha=0$, the SCF can also be interpreted as power spectral density of the signal. For other values of $\alpha, \mathrm{SCF}$ is cross-spectral density between the signal and the signal shifted in frequency by $\alpha$. So, if the signal being analyzed exhibits cyclostationarity, SCF will be non-zero for some $\alpha \neq 0$. Otherwise, only for $\alpha=0$ we will have non-zero values.

\section{Cyclostationary Properties of Resampled signals}

In the next section, we will employ the theory of cyclostationarity for finding the traces of geometric transforma- tions. To be able doing so, first we should show their periodically varying properties. To achieve this, we will assume the following simple, linear and stochastic model and assumptions:

$$
f(x)=(u * h)(x)+n(x)
$$

where $f, u, h, *$, and $n$ are the measured image, original image, system point spread function (PSF), convolution operator, and random variable representing the influence of noise sources statistically independent from the signal part of the image $(E\{n(x)\}=0)$. If we consider the first part of equation (7) to be deterministic, the covariance of equation (7) can be shown to be $R_{f}\left(x_{1}, x_{2}\right)=$ $\operatorname{Cov}\left\{f\left(x_{1}\right), f\left(x_{2}\right)\right\}=E\left\{\left(f\left(x_{1}\right)-\bar{f}\left(x_{1}\right)\right)\left(f\left(x_{2}\right)-\right.\right.$ $\left.\left.\bar{f}\left(x_{2}\right)\right)\right\}=\operatorname{Cov}\left\{n\left(x_{1}\right), n\left(x_{2}\right)\right\}=R_{n}\left(x_{1}, x_{2}\right)$, where $R_{f}$ is the covariance matrix of measured image $f(x)$, and $R_{n}$ is the covariance of random process $n(x)$.

By $f_{k}$ we will denote a discrete signal representing the samples of $f(x)$ at the locations $k \Delta, f_{k}=f(k \Delta)$, where $\Delta \in \mathcal{R}^{+}$, is the sampling step and $k \in \mathcal{N}_{0}$.

There are two basic steps in geometric transformations. In the first step a spatial transformation of the physical rearrangement of pixels in the image is done. Coordinate transformation is described by a transformation function, $T$, which maps the coordinates of the input image pixel to the point in the output image (or vice versa):

$$
x^{\prime}=T_{x}(x, y) \quad y^{\prime}=T_{y}(x, y)
$$

The second step is the interpolation step. Here pixels intensity values of the transformed image are assigned using a constructed low-pass interpolation filter, $w$. As the word interpolation signifies ${ }^{2}$, the interpolation process can be described using the following convolution:

$$
f^{w}(x)=\sum_{k=-\infty}^{\infty} f_{k} w\left(\frac{x}{\Delta}-k\right)
$$

The sinc function is hard to implement in practice because of its infinite extent. Thus, many different simpler interpolation kernels of bounded support have been investigated and proposed so far. We will be concerned with following low-order piecewise local polynomials: nearestneighbor, linear, cubic and truncated sinc. These polynomials are used extensively because of their simplicity and implementation unassuming properties. As will be shown, these interpolators bring noticeable periodic artifacts into the signal.

As pointed out in $[14,10]$, the covariance function of an

\footnotetext{
${ }^{2}$ The word "interpolation" originates from the Latin word "inter", meaning "between", and verb "polare", meaning "to polish" [11].
} 
interpolated signal is given by:

$$
\begin{aligned}
& R_{f^{w}}(x, x+\xi)= \\
& \quad \sum_{k_{1}=-\infty}^{\infty} \sum_{k_{2}=-\infty}^{\infty} w\left(\frac{x}{\Delta}-k_{1}\right) w\left(\frac{x+\xi}{\Delta}-k_{2}\right) R_{f}\left(k_{1}, k_{2}\right)
\end{aligned}
$$

If we assume constant variance random process, then the variance of $f^{w}, \operatorname{var}\left\{f^{w}(x)\right\}$, as a function of the position $x$ can be represented in the following way:

$$
\operatorname{var}\left\{f^{w}(x)\right\}=R_{f^{w}}(x, x)=\sigma^{2} \sum_{k=-\infty}^{\infty} w\left(\frac{x}{\Delta}-k\right)^{2}
$$

where $\sigma^{2}=R_{n}\left(k_{1}, k_{2}\right)$. This equation can be obtained if $R_{f}\left(k_{1}, k_{2}\right)$ has a short-range correlation [14]. Similarly, the covariance can be represented like:

$$
R_{f^{w}}(x, x+\xi)=\sigma^{2} \sum_{k=-\infty}^{\infty} w\left(\frac{x}{\Delta}-k\right) w\left(\frac{x+\xi}{\Delta}-k\right)
$$

Now, by assuming that $\vartheta$ is an integer, we can notice that

$$
\operatorname{var}\left\{f^{w}(x)\right\}=\operatorname{var}\left\{f^{w}(x+\vartheta \Delta)\right\}, \vartheta \in \mathcal{Z}
$$

Thus, $\operatorname{var}\left\{f^{w}(x)\right\}$ is periodic over $\mathrm{x}$ with period $\Delta$. We verify this in the following way:

$$
\begin{aligned}
\operatorname{var}\left\{f^{w}(x+\vartheta \Delta)\right\} & =\sigma^{2} \sum_{k=-\infty}^{\infty} w\left(\frac{x+\vartheta \Delta}{\Delta}-k\right)^{2} \\
& =\sigma^{2} \sum_{k=-\infty}^{\infty} w\left(\frac{x}{\Delta}-(k-\vartheta)\right)^{2} \\
& =\operatorname{var}\left\{f^{w}(x)\right\}
\end{aligned}
$$

Very similarly, it can be shown that the covariance of $f^{w}$, $R_{f^{w}}(x, x+\xi)$, is periodic in the same way.

\section{Detecting Cyclostationarity}

The previous section showed that resampled images contain cyclostationary features. Many efficient methods capable of detecting cyclostationary features $[5,15]$ have been proposed so far.

Theory of cyclostationarity has shown that a cyclostationary signal has a frequency spectrum that is correlated with a shifted version of itself [2]. Based on this, we focus on detecting the traces of cyclostationarity by estimating the spectral correlation function. To estimate the SCF, we can simply use equation (6). But, due to its computational complexity, instead of this, we rather use a more computationally effective SCF estimation method based on Fast Fourier Transform (FFT). FFT algorithm has computational complexity $\mathrm{O}\left(\operatorname{nlog}_{2} \mathrm{n}\right)$.
Let's say $f(x, y)$ is the image being analyzed and $F(n, u)$ is a matrix containing FFT of image's rows (i.e., $F(1, u)$ contains the one-dimensional FFT of the first row of $f(x, y)$ ). The SCF can be estimated in the following way:

$$
S_{f}^{\alpha}(u)=\frac{1}{N} \sum_{n=0}^{N-1} F_{n, u} \cdot F_{n, u+\alpha}^{*},
$$

where $*$ denotes complex conjugate and $N$ is the number of image's rows.

Data obtained can be combined together to create the resulting correlation map:

$$
\rho_{f}(\alpha)=\sum_{u}\left|S_{f}^{\alpha}(u)\right|^{2}
$$

To demonstrate the method's output obtained using equation (16), we apply it to several images resized by various scaling factors, see Figure 1. Here, to get clear peaks, columns of $F(n, u)$ were scaled to have values between 0 and 1. As apparent from Figure 1, cyclostationary features resulting from the scaling operation are exhibited by distinctive peaks.

\subsection{Adaptation of the Proposed Method}

The method so far presented works well (produces clear peaks) when the scaling rate is big enough to introduce strong correlation into the image. When the image is transformed by a lower scaling rate, the cyclostationary features are not strong enough to be detectable using the basic method (see Figure 1 (c)). This drawback can be overcome using a traditional way based on passing the analyzed image through a set of band-pass filters. We use a set of derivative filters as band-pass filters. If $f(x, y)$ denotes the image being analyzed, then $\mathbf{d}^{\mathbf{n}}$ is a band-passed image containing the horizontal and vertical derivative approximations:

$$
\mathbf{d}^{\mathbf{n}}=d_{h}^{n} * d_{v}^{n} * f(x, y),
$$

where $\mathbf{n}$ denotes the order of the derivative filter. The following first order horizontal and vertical derivative filters formed our filters for derivative approximations:

$$
d_{h}^{1}=\left[\begin{array}{ll}
-1 & 1
\end{array}\right], \quad d_{v}^{1}=\left[\begin{array}{c}
-1 \\
1
\end{array}\right]
$$

Using these filters we get significantly more accurate and robust outcomes. After performing various experiments with resized images of different structures, content, brightness and noise characteristics we achieved good results using only a lower and a higher derivative filter. The proposed method works by seperately applying equation (15) using $\mathrm{d}^{\mathbf{1}}$ and $\mathbf{d}^{\mathbf{5}}$ :

$$
\begin{aligned}
& \rho_{\mathbf{d}^{1}}(\alpha)=\sum_{u}\left|S_{\mathbf{d}^{1}}^{\alpha}(u)\right|^{2} \\
& \rho_{\mathbf{d}^{5}}(\alpha)=\sum_{u}\left|S_{\mathbf{d}^{5}}^{\alpha}(u)\right|^{2}
\end{aligned}
$$


(a)

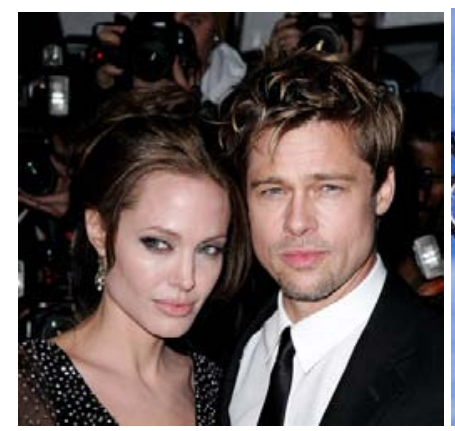

(d)

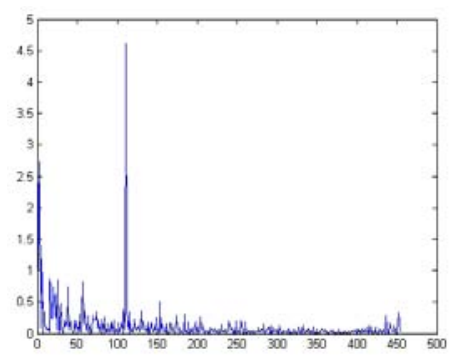

(b)

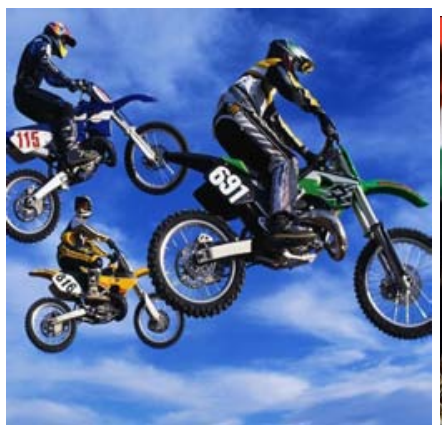

(e)

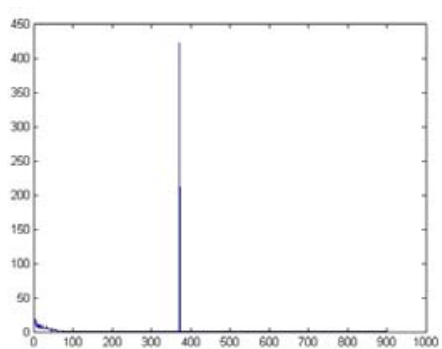

(c)

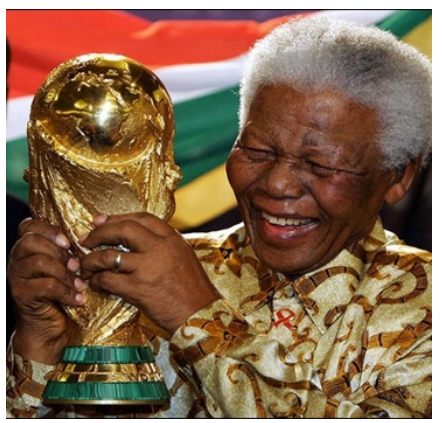

(f)

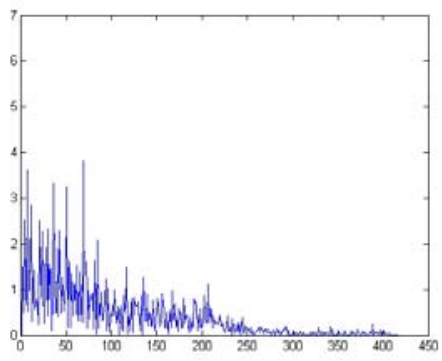

Figure 1. Each column shows an example image and its corresponding correlation map obtained by application of equation (16) to the resized version of this image. The used scaling rates were (from left to right) 1.32. 1.7 and 1.2. In all cases the bicubic interpolation has been used. The distinctive peaks signify the resizing procedure. The application of the basic method to Figure 1 (c) failed.

(a)

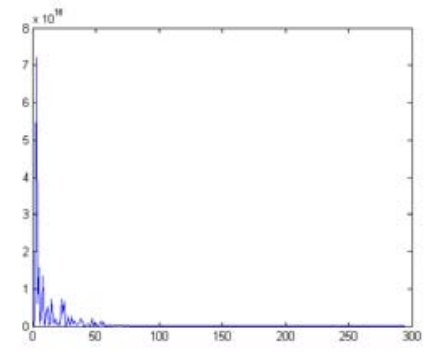

(d)

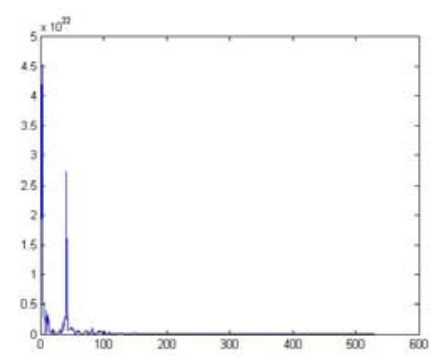

(b)

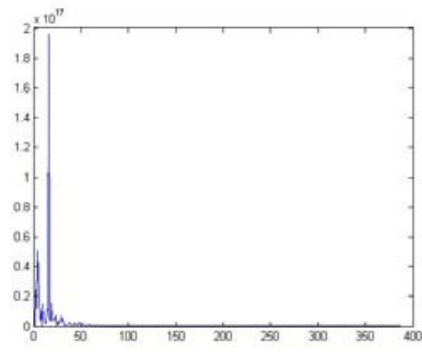

(e)

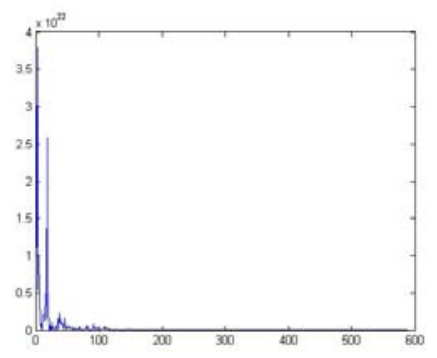

(c)

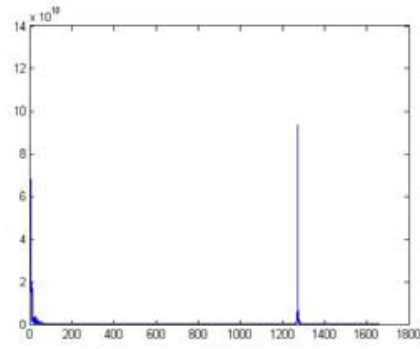

(f)

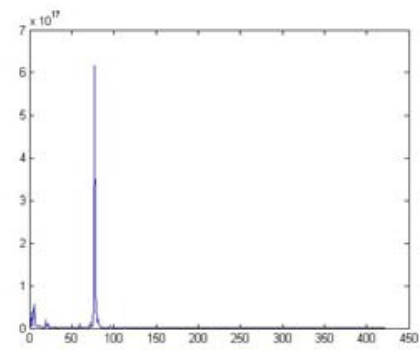

Figure 2. A few examples of the method's output. In (a) the output of the image applied to the non-resized version of Figure 1 (a) is shown. As apparent, there is no clear peak. (b) and (c) show the output of the method for resized Figure 1 (a) with scaling rates 1.04 and 4.27 , respectively. (d) and (e) show the output of the method for resized Figure 1 (b) with scaling rates 0.93 and 1.03 . In (f) the output of the method applied to the scaled version of Figure 1 (c) with scaling rate 1.22 is shown. In all cases the bicubic interpolation has been used.

If the image being analyzed has been geometrically transformed, then at least one of the correlation maps $\rho_{\mathbf{d}^{1}}(\alpha), \rho_{\mathbf{d}^{5}}(\alpha)$ will exhibit a detectable peak (see Figure
2). In all examples, the method has been applied to the green color band.

Detected peaks are directly related with the scaling rate. 


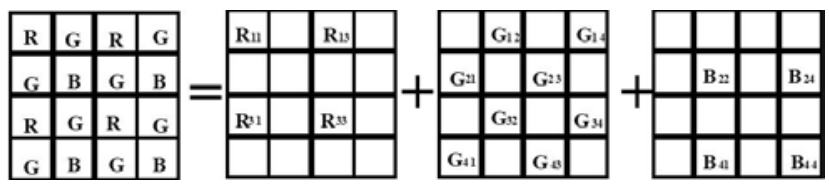

Figure 3. Decomposition of the Bayer CFA into three sub-sampled lattices.

So, using the position of the occurred peak, the particular scaling rate can be estimated. For example, when assuming the upsampling operation and $a$ will denote the position of the peak in interest obtained by applying the method to $f(x, y)$, then the scaling rate can be estimated in the following way: $s=\frac{y}{y-a}$.

\subsection{Other Geometric Transformations}

The method proposed so far works only for scaling. There are several ways how to apply it to other geometric transformations. For example, when dealing with rotation, it is possible to apply the method in various directions. The direction which includes strong cyclostationary features will exhibit with strong peaks in the method's output. This idea works not only for rotation, but also for general affine transformations.

\section{Influence of Color Filter Array Interpola- tion and JPEG}

As color filter array (CFA) interpolation and JPEG compression bring into the image a specific periodicity, it could be interesting to show their influence on method's output.

\subsection{CFA Interpolation}

Many digital cameras are equipped with a single charge coupled device (CCD) or complementary metal oxide semiconductor (CMOS) sensor. At each pixel location only a single color sample is captured. The color images are typically obtained in conjunction with a CFA [8]. The most commonly used CFA is called Bayer CFA after the name of its inventor ${ }^{3}$. As shown in Figure 3, it consists of alternating red and green pixels on odd lines and green and blue pixels on even lines. Missing colors are computed by an interpolating process, called CFA interpolation. There are many CFA interpolation algorithms leading to different results (bilinear, bicubic, median-based, gradient-based, SHT, adaptive, directional filtering, etc.). Some of these interpolation methods bring into the image a strong periodic correlation (for example, bilinear CFA).

Let's assume the most simplest CFA - the bilinear. Here to obtain the red pixel $R$ at position $(2,2)$ and position $(3,2)$ in the grid (see Figure 3) the following equations are used:

$$
\begin{aligned}
R_{2,2} & =\frac{R_{1,1}+R_{1,3}+R_{3,1}+R_{3,3}}{4} \\
R_{3,2} & =\frac{R_{3,1}+R_{3,3}}{2}
\end{aligned}
$$

As apparent, pixels of bilinear CFA image are perfectly periodically correlated to their neighboring pixels. Thus, we can expect in the output of the method a peak corresponding to this interpolation while the image being analyzed was not resized. Output of the method applied the non-resized bilinear CFA reconstructed version of Figure 4 (a) is shown in Figure 4 (b).

Figure 4 (c) shows the output of the method to the resized version (scaling rate 1.25) of the bilinear CFA image shown in Figure 4 (a). As apparent, the peak corresponding to the scaling transformation is clear and traces of CFA interpolation are defeated. Figure 4 (d) shows the output of the image applied to non-resized Figure 4 (a) reconstructed using a more today CFA interpolation method [12] based on directional filtering. As apparent, the traces of CFA interpolation have no false-positive effect on the method's output. Experiments of this section were carried out using the red color band.

The early CFA interpolation methods (for example, bilinear) which bring into the image perfect periodic correlation may cause false peaks. Fortunately, these methods are not commonly used in digital cameras. (a)

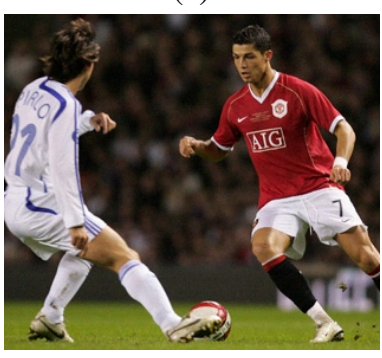

(c)

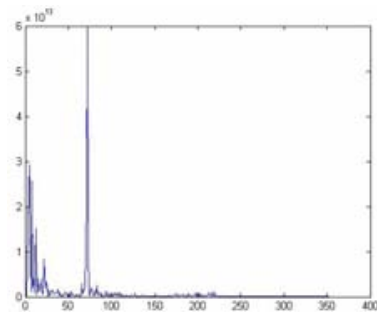

(b)

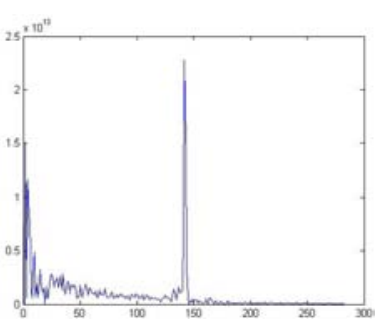

(d)

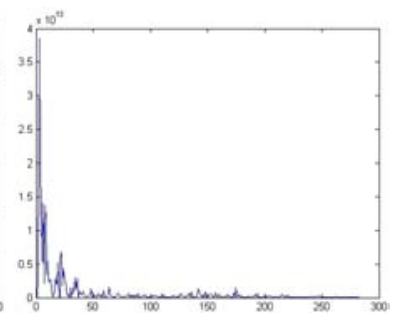

Figure 4. Effect of CFA interpolation. (a) shows the analyzed image. In (b) non-resized bilinear, (c) resized bilinear CFA (scaling rate 1.25) and (d) non-resized directional filtering CFA are shown.

\footnotetext{
${ }^{3}$ Doctor B.E. Bayer from Eastman Kodak
} 
Table 1. Detection accuracy [\%]. Each cell corresponds to the average detection accuracy from 1000 images.

\begin{tabular}{|c||c|c|c|c|c|}
\hline scaling rate & 0.75 & 0.80 & 0.85 & 0.90 & 0.95 \\
\hline accuracy & 25 & 90 & 92 & 98 & 100 \\
\hline \hline scaling rate & 0.97 & 1.00 & 1.03 & 1.05 & 1.10 \\
\hline accuracy & 100 & 95 & 100 & 100 & 100 \\
\hline \hline scaling rate & 1.20 & 1.40 & 1.50 & 1.60 & 1.70 \\
\hline accuracy & 100 & 100 & 100 & 100 & 100 \\
\hline \hline scaling rate & 1.80 & 1.90 & 2.00 & 2.10 & 2.20 \\
\hline accuracy & 100 & 100 & 100 & 100 & 100 \\
\hline
\end{tabular}

\subsection{JPEG Compression}

As it is well-known, the JPEG compression technique divides the image into $8 \times 8$ pixel blocks to which discrete cosine transform (DCT) based coding is applied. This blocking artifact brings into the image a periodicity producing peaks at method's outputs at positions $\frac{1}{8} y \cdots \frac{7}{8} y$. This effect is visible by applying the method to JPEG compressed images with quality factor 90 and lower. Of course, the visibility of peaks also depends on image's characteristics.

\section{Quantitative Experiments}

The method has been applied to 1000 images undergone various scaling transformations. The size of the investigated images was $512 \times 512$ pixels. All experiments were carried out in Matlab. In all cases never-compressed images have been used. Table 1 shows the detection accuracy of the method applied to bicubic resized images. The detection accuracy expresses the success of the method in expressing the interpolation by a clear and easily detectable peak either in $\rho_{\mathbf{d}^{1}}(\alpha)$ or $\rho_{\mathbf{d}^{5}}(\alpha)$. Note that the detection is nearly perfect for scaling rates greater than 0.90 . Here, the amount of the cyclostationary features is strong enough to be detectable. When the image is downsampled, the power of cyclostationary features brought into the signal is weakened and a lot of information is lost (due to downscaling). This makes the detection of downsampling difficult. Shown statistics for scaling rate 1 (non-resized) corresponds to the false positives rate of the method. Here, if no peaks are found, the image is denoted as non-resized.

\section{Conclusions and Further Research}

Results obtained are promising and show that employing cyclostationarity methods can be effective in many applications in computer vision and pattern recognition. Further research might explore application and evaluation of various types of cyclostationary feature detection methods, use of filter banks and extension to other geometric transformations.

\section{Acknowledgements}

This work has been supported by the Czech Science Foundation under the project No. GACR 102/08/0470.

\section{References}

[1] A. C. Gallagher. Detection of linear and cubic interpolation in jpeg compressed images. In $C R V$ '05: Proceedings of the The 2nd Canadian Conference on Computer and Robot Vision (CRV'05), pages 65-72, Washington, DC, USA, 2005. IEEE Computer Society.

[2] W. A. Gardner. The spectral correlation theory of cyclostationary time-series. Signal Process., 11(1):13-36, 1986.

[3] W. A. Gardner. Statistical spectral analysis: a nonprobabilistic theory. Prentice-Hall, Inc., Upper Saddle River, NJ, USA, 1986.

[4] W. A. Gardner. Exploitation of spectral redundancy in cyclostationary signals. IEEE Signal Processing Magazine, 8(2):14-36, April 1991.

[5] W. A. Gardner, A. Napolitano, and L. Paura. Cyclostationarity: Half a century of research. Signal Processing, 86(4):639-697, 2006.

[6] M. Johnson and H. Farid. Exposing digital forgeries by detecting inconsistencies in lighting. In ACM Multimedia and Security Workshop, New York, NY, 2005.

[7] M. Kirchner. Fast and reliable resampling detection by spectral analysis of fixed linear predictor residue. In Proceedings of the 10th ACM workshop on Multimedia and security, pages 11-20, New York, NY, USA, 2008. ACM.

[8] S.-H. Lam and C.-W. Kok. Demosaic: Color filter array interpolation for digital cameras. In PCM '01: Proceedings of the Second IEEE Pacific Rim Conference on Multimedia, pages 1084-1089, London, UK, 2001. Springer-Verlag.

[9] B. Mahdian and S. Saic. Detection of copy-move forgery using a method based on blur moment invariants. Forensic science international, 171(2-3):180-189, 2007.

[10] B. Mahdian and S. Saic. Blind authentication using periodic properties of interpolation. IEEE Transactions on Information Forensics and Security, 3(3):529-538, September 2008.

[11] E. Meijering. A chronology of interpolation: From ancient astronomy to modern signal and image processing. Proceedings of the IEEE, 90(3):319-342, March 2002.

[12] D. Menon, S. Andriani, and G. Calvagno. Demosaicing with directional filtering and a posteriori decision. IEEE Transactions on Image Processing, 16(1):132-141, 2007.

[13] A. Popescu and H. Farid. Exposing digital forgeries by detecting traces of re-sampling. IEEE Transactions on Signal Processing, 53(2):758-767, 2005.

[14] G. Rohde, C. Berenstein, and D. Healy. Measuring image similarity in the presence of noise. Proceedings of the SPIE Medical Imaging: Image Processing, 5747:132-143, February 2005.

[15] E. Serpedin, F. Panduru, I. Sari, and G. B. Giannakis. Bibliography on cyclostationarity. Signal Process., 85(12):22332303, 2005. 